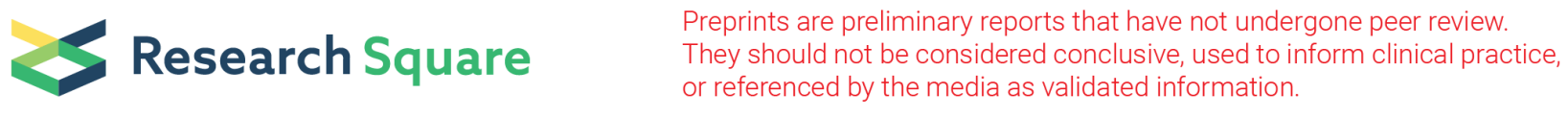

\title{
Glycation Markers in the Geriatrics Patients
}

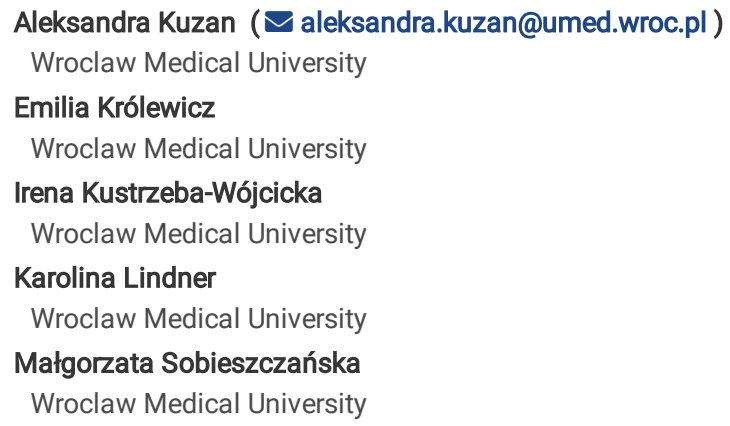

\section{Research Article}

Keywords: aging, geriatric care, elderly diseases, diabetes mellitus type 2, glycation markers

Posted Date: April 30th, 2021

DOI: https://doi.org/10.21203/rs.3.rs-448168/v1

License: (c) (i) This work is licensed under a Creative Commons Attribution 4.0 International License. Read Full License 


\section{Abstract}

Background: Medical care for geriatric patients is a great challenge, mainly due to overlapping various deficits relevant to physiologic ageing with numerous coexisting diseases, of which the most common are diabetes mellitus and atherosclerosis. In case of diabetes, glycation process is intensified, which accelerates atherosclerosis development and diabetic complications. Our goal was to investigate the relationship between the classical biochemical parameters of diabetes and atherosclerosis (fasting glucose, glycated hemoglobin (HbA1c), low and high density lipoproteins (LDL, HDL), triglycerides, etc.), as well as parameters which may indicate a nephropathy (creatinine, glomerular filtration rate - GFR), and the parameters strictly related to glycation. Methods: We analyzed the patients' serum concentration of fluorescent glycation products, concentration of soluble receptor for advanced glycation products (sRAGE), lipoprotein receptor-1 (LOX-1), galectin 3 (GAL3), scavenger receptor class A (SR-A) and scavenger receptor class B (SR-BI), as well as the level of lipid peroxidation and free amine content. Results: Among the identified correlations, the most interesting are the following : sRAGE with triglycerides ( $r=0.47)$; sRAGE with SR-BI ( $r=0.47)$; SR-BI with LOX-1 ( $r=0.31)$, and SR-BI with HDL $(r=-0.30)$. It has been shown that pentosidine and fluorescent AGEs as well as reactive free amine contents are significantly higher in elderly patients with ischemic heart disease. Fluorescent AGEs and pentosidine are also significantly higher in patients with arterial hypertension. The influence of diabetes therapy on parameters related to peroxidation and glycation was also analyzed.

Malondialdehyde turned to be higher in patients with diabetes mellitus type 2 not treated with insulin or metformin than in those treated with both medications $(p=0.052)$. GAL3 was found to be lower both in persons without diabetes and in diabetics treated with metformin ( $p=0.005)$. LOX1 was higher in diabetic patients not treated with metformin or insulin, and lowest in diabetics treated with both insulin and metformin, with the effect of metformin reducing LOX1 levels $(p=0.039)$. Conclusions: Our results were the basis for a discussion about the diagnostic value in clinial practice of LOX-1 and GAL3 in geriatric patients with diabetes and also provide grounds for inferring the therapeutic benefits of insulin and metformin treatment.

\section{Introduction}

In 1909, the Viennese doctor, Ignatz Leo Nascher, introduced the term "geriatrics" which meant "old age medicine" [1]. As a pioneer of this clinical discipline, he justified the need to separate getiatrics from adult medicine [2]. In 2004, the World Health Organization (WHO) defined geriatrics as a field of medicine dealing with diseases and healthcare for the elderly [3].

The number and proportion of people aged 60 years and older in the global population is increasing. In 2019 , the number of elderly people was 1 billion, and this number will increase to 1.4 billion by 2030 and 2.1 billion by 2050 [4]. This increase is occurring at an unprecedented pace and will accelerate in coming decades, particularly in developing countries. The explanation for this phenomenon is mainly the improvement of life expectancies, high quality of healht care, as well as a post-World War 2 baby boom [4] and declining total fertility rates [5]. Seniors suffer from functional and mental decline, cognitive deficits, frailty, multiple comorbidities, resulting, as a consequence, in polypharmacy. That is why it is so important to develop geriatrics as an interdisciplinary and multidimensional field of medicine using a comprehensive approach to elderly patients.

Clinical manifestations of diseases affecting elderly persons include a lot of somatic and mental problems. This applies to atherosclerotic cardiovascular disease, diabetes, arterial hypertension, osteoarthritis, urinary incontinence, hearing problems, visual impairment as well as osteoporosis and sarcopenia. Also, dementia, including Alzheimer disease and depression, cannot be omitted [6]. Furthermore, there is the association between chronic low-grade inflammation ("inflammaging") and many adverse health outcomes inelderly patients, including chronic diseases, functional decline and mortality [7].

Diabetes mellitus (DM), in particular type 2 diabetes (T2DM), is the most common disease in the older adults . It is estimated that T2DM affects up to 40 percent of people over 65 years old in developed and even in developing countries [8]. Approximately, 20 percent of older persons suffer from clinically apparent DM, and a similar proportion have undiagnosed DM [9]. Fueled in part by the obesity epidemic, this number is projected to double in the next 20 years and quadruple by 2050 [10]. Diabetes, especially nowadays, is a huge threat to older people, because of the pandemic related to the emergence of SARS-CoV-2 and COVID-19 As a result the worse access to health care and physicians and the lack of physical activity, associated with being closed at home, are observed. Theee factors contribute to carbohydrate metabolism disorders and insufficent glycemic control [11, 12]

Chronically sustained high blood sugar levels lead to damage and abnormal function of many organs - especially, the heart, blood vessels, kidneys, nerves, and eyesight [13],[14]. Diabetes mellitus is associated with an increased level of free radicals, disturbances of the enzymatic antioxidant defense system and lower concentration of exogenous antioxidants. In consequence, these abnormalities lead to a redox imbalance called oxidative stress [15].

In diabetes mellitus, the theory of oxidative stress is associated with autooxidation of glucose (glycoxidation), which produces reactive ketoaldehydes. This intensifies the process of non-enzymatic protein glycation leading to irreversible glycation end productscalled AGEs. During this process, due to the strong dependence on oxygen, many toxic oxygen derivatives are formed. In people with diabetes, the serum concentrations of pentosidine, pyraline or carboxymethyl lysine are increased. The severity of the production of reactive oxygen species (ROS) depends on the degree of protein glycation, and thus on glycemia. Many proteins in the body undergo the process of glycation, including proteins of the basement membrane and blood proteins [16].

In the present study, a focus was placed not only on AGEs but also on their receptors. Glycation products are ligands for numerous proteins, the most important of which appear to be: Receptor for Advanced Glycation End Products (RAGE; synonym: AGER); scavenger receptors class A (SR-A; synonyms: MSR1, SCARA1, Macrophage acetylated LDL receptor I and II, Scavenger receptor class A member 1), and class B, type I (SR-BI; synonyms: SCARB1, CD36L1; SRB1; CLA1; Collagen Type I Receptor Thrombospondin Receptor-Like 1; CD36 and LIMPII analogous 1); Lectin Like Oxidized Low Density Lipoprotein Receptor 1 (LOX-1), and galectin 3 (GAL3) [17-21].

Gaining detailed knowledge about the factors leading to the aging process and the course of diseases occurring in the elderly (including diabetes) makes it possible to develop a way to delay the decline in the functionality of the human body and at the same time extend life. 


\section{Materials And Methods}

The research material were serum samples taken during hospitalization at the Department of Geriatrics, University Teaching Hospital in Wrocław. Totally, seventynine patients who were qualified for the scientific project gave written permission for participation in the study and taking biological material. The Bioethical Commission at Wroclaw Medical University gave written permission (opinion number KB-344/2017). The results of routine laboratory tests, like serum levels of: glucose, total protein, hemoglobin, glycated hemoglobin (HbA1c), C-reactive protein (CRP), cholesterol, HDL, LDL, triglycerides, creatinine, and uric acid, as well glomerular filtration rate (GFR) were used for statistical analyses. Forty one diabetic patients were assessed in four groups: (1) treated with metformin only, (2) treated with insulin only, (3) treated with both medications, and (4) treated with other hypoglycemic drugs (glimepiridum, gliclazide, linagliptin, liraglutide). The characteristics of the study population are presented in Table 1.

\section{Analysis of fluorescent AGE and pentosidine content using fluorometric method}

Pentosidine is a ribose-derived glyco-oxidation product of arginine and lysine residues and is one of the fluorescent forms of AGE. The methodology of pentosidine and total pool of fluorescent AGEs determination was a slight modification of that of Leszek et al. (Leszek et al., 2005). Serum samples were diluted 100 -fold with $0.9 \% \mathrm{NaCl}$. The absorbances of these samples at $\lambda=280 \mathrm{~nm}$ were measured. The fluorescence of samples at excitation wavelength 370 $\mathrm{nm}$ and emission $400 \mathrm{~nm}$ were measured to assess the content of total fluorescent AGEs. To assess the total pentosidine content in the sample, the fluorescence of the samples was measured at excitation wavelength $335 \mathrm{~nm}$ and emission $385 \mathrm{~nm}$. Fluorescence was divided by Abs 280 assuming the resulting values as data expressed in arbitrary units.

\section{Assessment of content of compounds with thiol groups (glutathione)}

Determination of the glutathione (one of main factors responsible for the antioxidative mechanism in the body) concentration is usually performed as a measure of thiol groups content using Ellman's reagent (5,5'-dithio-bis-(2-nitrobenzoic acid); DTNB). The method was developed in 1991 by Diplock [23] et al. To achieve this goal, a reaction between compounds containing thiol groups from patients' serum and 0,1M DTNB in 10mM sodium-phosphate buffer pH 8.00 was conducted with the presence of $10 \%$ SDS. Parallel to this, an analogical reaction with glutathione of concentration $0.25-1 \mathrm{mM}$ was performed in order to draw a standard curve. The reaction was conducted for 60 minutes at $37^{\circ} \mathrm{C}$. In the next step, an absorbance against blank sample at $\lambda=412 \mathrm{~nm}$ was measured, and on the basis of data from the standard curve, the content of thiol group was calculated in examined material.

\section{Assessment of lipid peroxidation on the basis of determination of malondialdehyde (MDA) in serum}

A method of estimation of lipid peroxidation intensity is based on reaction of malondialdehyde with thiobarbituric acid (TBA). The pink product obtained was later determined by spectrophotometry [24].The reactive mixtures consisted of serum, 15\% TCA acid solution in $0.25 \mathrm{M} \mathrm{HCl}$ and $0.37 \% \mathrm{TBA}$ in $0.25 \mathrm{M} \mathrm{HCl}$. The mixtures were incubated for 20 minutes at $100^{\circ} \mathrm{C}$, then cooled down, rotated for 5 minutes at $4700 \mathrm{RPM}$. Absorbance of supernatant was measured at 535 $\mathrm{nm}$. Calculation of MDA concentration was performed using the Lambert-Beer law, at absorbance coefficient $\varepsilon=156 \mathrm{mmol}{ }^{-1} \cdot \mathrm{L} \cdot \mathrm{cm}^{-1}$.

\section{Reactive free amine content}

The procedure is described in the work of Fracasso et al. [25]. Serum samples were diluted 200-fold with $50 \mathrm{mM}$ carbonate buffer (pH 10.5). Then, 9- $\mu \mathrm{L}$ aliquots of the diluted specimens were pipetted into a 96 -well microplate and $91 \mu \mathrm{L}$ of freshly made o-phthalaldehyde (OPA) solution (5 mg OPA, $100 \mu \mathrm{L}$ pure ethanol, $5 \mu \mathrm{L} \beta$-2-mercaptoethanol, and $10 \mathrm{~mL} 50 \mathrm{mM}$ carbonate buffer $\mathrm{pH} 10.5$ ) was added in all wells. Samples were read immediately at $340 \mathrm{~nm}$ excitation and $455 \mathrm{~nm}$ emission wavelengths.

\section{Analysis of GAL3 and LOX1 protein content}

The concentration of GAL3 and LOX1 in serum samples was performed using human immunoassay ELISA kits from Elabscience®. The manufacturer's instructions were followed.

\section{Analysis of sRAGE, SCRAB1 and MSR1 protein content}

The concentration of sRAGE, SCRAB1 and MSR1 in serum samples was performed using human immunoassay ELISA kits from ELK Biotechnology. The manufacturer's instructions were followed.

\section{Statistical analysis}

Statistical analysis was conducted using the data analysis software system Statistica (version 13.3, StatSoft). Frequency analysis was conducted using Fisher's exact test or $\chi 2$ test. For comparison of all the variables between groups (with diabetes/ without diabetes; with hypertension/ without hypertension; with ischemic heart disease (IHD) / without IHD; etc. ), the Mann-Whitney U test was used. Kruskal-Wallis test with post-hoc median test were used when comparing more than two continuous variables. All calculated p-values where two-sided, and statistical significance level was set at $p<0.05$. Spearman rank correlation analysis was used to determine associations between oxidative and inflammatory markers with selected parameters. To determine the most important predictors of AGE, Pentosidine and SR-BI levels, modelling with Generalized Linear Models (GLM) was used. As covariates in the models, all those variables which showed significant relatshionship with the dependant variable were introduced in the univariate analysis. The variables were fitted using Gaussian distribution with the identity link function. The models were constructed in a two-way step procedure based on the Wald test results with the significance level increased to $20 \%$ and Akaike information criterion (AIC). Statistics for the variables rejected during modeling, which are given in the tables, were obtained after their independent inclusion in the final model. 


\section{Results}

1. Correlations between variables

Table 2 shows what correlations are found between the analyzed parameters. Due to their number, not all of them are listed, but only those that seem to be the most interesting and important, i.e. have the highest $r$-factor at $p<0.005$ and are relatively little or not described in the literature on the subject, are emphasized, as given below:

Galectin 3 content strongly correlates with BMI $(r=0.62, p<0,0001)$, as well as with glucose $(r=0.39, p<0,0001)$, with triglycerides $(r=0.36, p=0,002)$, and negatively with GFR $(r=-0.32, p=0,006)$.

The content of fluorescing AGEs positively correlates with pentosidine $(r=0.67, p<0,0001)$ and creatinine $(r=0.45, p<0,0001)$, and negatively with GFR ( $r=-0.48$, $p<0,0001)$.sRAGE content correlates with triglycerides $(r=0.47, p=0,009)$ and $S R-B I(r=0.47, p=0,013)$

SR-BI content positively correlates with LOX1 $(r=0.32, p=0,013)$ and negatively with HDL $(r=-0.30, p=0,02)$.

Glutatnion and SR-A did not correlate with any parameters.

2. Relationships between diseases and biochemical parameters

Fluorescent AGEs and pentosidine have been shown to be higher in people with ischemic heart disease (IHD) ( $p=0.0124, p=0.0018$, respectively) (Fig. 1 AB). Likewise, OPA is higher in people with IHD ( $p=0.0491)$ (Fig. 1E). It was also shown that hypertensive patients have significantly elevated levels of fluorescent AGEs and pentosidine ( $p=0.050, p=0.003$, respectively) (Fig. 1CD).

None of the analyzed parameters were significantly associated with the history of myocardial infarction, but one of them, namely OPA, was significantly increased in post-stroke patients $(p=0.009)$ (Fig. 1F).

3. Influence of diabetes therapy on parameters related to glycation and peroxidation

Serum GAL3 content is found to be lower in nondiabetic subjects $(\mathrm{p}=0.0047)$, and in the diabetic group it is lower in subjects treated with metformin as compared with diabetics treated with insulin or treated with drugs other than metformin and insulin ( $p=0.0268)$ (fig. $2 A$ i B).

LOX1 is high in diabetic patients not treated with insulin or metformin and statistically significantly lower in people treated with both insulin and metformin compared to people treated with insulin alone ( $p=0.0398)$ (Fig. 2 C). The median for the result of diabetics treated separately with insulin and separately with metformin is lower than for those not treated with insulin or metformin; probably due to the small number of patients not treated with insulin or metformin, the statistical significance was not obtained here.

MDA is higher in diabetic patients not treated with insulin or metformin than those treated with metformin and insulin simultaneously ( $p=0.052$ ) (Fig. 2D). The median for those treated with one drug is also lower than for those not treated, but statistical significance was also not obtained here.

4. Statistical modeling results

In the multivariate analysis of SR-BI level based on HDL and uric acid, as the variables, only HDL turned out to be a significant predictor (Wald $\chi 2$ 6.60; $\mathrm{df} 1$; $\mathrm{p}=0.01)$.

In the multivariate analysis of AGE levels on the basis of variables IHD, GFR and creatinine, the important predictors were IHD and GFR (Wald $\chi 2$ 4.69; $\mathrm{df} 1$; $p=0.03$ and Wald $\chi 212.126 ; \mathrm{df} 1 ; p=0.00$, respectively).

The following variables were used in the multivariate analysis of pentosidine levels: IHD, hypertension, GFR, creatinine and HbA1C. The best model included three predictors: IHD, hypertension and GFR, of which the first two were barely significant (IHD Wald $\chi 2$ 3.911; df 1; $p=0.048$; hypertension Wald $\chi 2$ 3.563; df 1 ; $p=0.59 ;$ GFR 1.644 df $1, p=0.2$ ).

\section{Discussion}

Galectin-3 is a protein from the group of $\beta$-galactoside binding lectins with pro-inflammatory properties, presented as a novel biomarker of pathological conditions, such as various types of cancer, especially thyroid cancer [17], acute and chronic heart failure [26, 27], chronic pancreatitis [18], prediabetes state and apparent diabetes [28], depression [29] and many others. It has multiple functions including cells adhesion, inflammation, and extracellular matrix formation, differentiation, proliferation, embryogenesis, and host-pathogen interactions [29-31]. As a lectin, it binds carbohydrates but it is characterized by a wide range of ligands and, in addition to simple sugars and disaccharides such as galactose, lactose or N-acetyllactosamine, it also binds large carbohydrate molecules, such as cell surface $\mathrm{N}$-glycans and advanced glycation products that are intensely generated during hyperglycemia [17, 18]. Moreover, the protein is found to cause cellular and systemic insulin resistance [31]. Therefore, we expected that galectin-3 would also reflect the various pathological states typical for the geriatric population.

The correlation of galectin 3 with BMI values has been demonstrated repeatedly in different study groups [30-32]. Our study confirmed this result. Similar correlations with glucose and triglycerides have been previously described, and these relationships are especially often observed in women with polycystic 
ovary syndrome and pregnant women with preeclampsia [32,33]. These correlations were also described based on studies on cardiac patients [27] and with hypothyroidism [34]. We describe these relationships probably for the first time for geriatric persons.

According to our observations, galectin-3 inversely correlates with GFR, indicating a relationship with kidney damage. Many studies have already concluded that galectin-3 strongly affects this organ's fibrosis and it has even been suggested that its measurement will allow to predict renal failure and patients' survival [35, 36].

Moreover, galectin 3 affects fibrosis of many other organs, including the heart after myocardial infarction (MI), hence the need to lower the level of this glycoprotein in order to avoid heart failure after MI [37]. Our studies show that metformin is a drug that is effective in reducing plasma GAL3 levels. Our results are consistent with the results of Asensio-Lopez et al [37]. The practical conclusion is that the administration of metformin in geriatric patients is beneficial not only in regulating carbohydrate metabolism but also in reducing the likelihood of heart failure threatening elderly persons.

In our study, galectin 3 is significantly higher in elderly patients with diabetes type 2. It would seem that galectin 3 is a very interesting diagnostic tool, and many authors present it as a novel biomarker, np. Alves, Hrynchyshyn, Kanukurti, King [26, 27, 29, 32]. However, doubts as to its diagnostic value are raised by the multitude of pathological conditions associated with an elevated level of this protein. When determining its elevated level, the geriatricians cannot state whether the cause is diabetes, heart failure, pancreatitis, cancer or depression. It seems that this parameter is not specific, thus is of little diagnostic value.

The second of the analyzed receptors for AGE, i.e., receptor for advanced glycation end products (RAGE), is the protein responsible for inflammation, apoptosis, reactive oxygen species (ROS) signaling, proliferation and autophagy [20]. SRAGE is a soluble form of protein that circulates in blood plasma competing for ligand, thus having RAGE-reversing, anti-inflammatory properties [18,38]. We expected a correlation between sRAGE and a fluorescent AGE, pentosidine, glucose or HbA1c. These expectations have not been met but it turned out that SRAGE correlates with triglycerides and SR-BI in our study population. Both of these parameters are related to lipid metabolism, TAG obviously, SR-BI due to the fact that, in addition to AGE, it also binds HDL, mediating selective uptake of cholesterol ether and HDL-dependent cholesterol efflux [19]. So far, no one has associated SR-BI and sRAGE. Only Marshe et al, who found that SRAGE is related to another scavenger receptor CD36 and blocks CD36 -mediated uptake of LDL modified by hypochlorous acid and in this way reduces foam cell formation [38]. Thus, it appears that both SRAGE and SR-BI have an inhibitory effect on the development of atherosclerosis and it is beneficial for the patient if the levels of these proteins, due to their mutual correlation, are high.

In our study group, the negative correlation between SR-BI and HDL was also observed. This is an expected result, as it has been proven that elevating SR-BI expression causes increase in HDL clearance, reduction in plasma HDL levels, and increase in biliary cholesterol levels [19].

The analysis revealed also that SR-BI correlates with one more parameter - Lectin-type oxidized LDL receptor 1 (LOX-1). In the latest publications (2020 and 2021), LOX-1 is presented as a promising target for early diagnosis and cardiovascular risk prediction [39-41]. Such enthusiastic announcements made us expect to find higher levels of LOX-1 in people with stroke, after a myocardial infarction or with obesity, but no such association was found in our geriatric population. Perhaps due to a small enough study group or too high degree of atherosclerosis in the entire study group. The correlation found between LOX-1 and SR-BI is, yet, a novelty in the subject literature and it is worth continuing research on the cause-and-effect relationship between these two receptors, since they correlate with each other.

Through modeling, we found that for SR-BI, HDL is a good predictor. West reported this relationship for subjects with hyperalphalipoproteinemia [42]. This is the first time that this dependence is described for the geriatric population. However, this finding is not clinically valuabl, because it is still more convenient to measure HDL levels in patients' blood serum .

Numerous data from in vitro experiments indicate that metformin inhibits expression of LOX-1. This is evidenced, for example, by the data of Hung et al. who studied this dependence in human umbilical vein endothelial cells (HUVECs) [43] or Shiu et al. who examined the relationship on human aortic endothelial cells incubated with AGE-BSA [44], and Ouslimani et al. who investigated bovine aortic endothelial cells [45]. The above-mentioned studies showed that metformin protects against oxLDL-induced endothelial apoptosis, oxLDL-induced intracellular calcium rise and mitochondrial dysfunction [43] as well as reduces, in dose-dependent manner, expression of LOX-1 both in stimulated (by either glucose or AGE) and in unstimulated cells [45]. No study of this relationship has been found in patients' trials, thus we are probably the first to report it. Our research shows, however, that treatment with metformin alone does not cause a large difference in the concentration of LOX-1 in the serum, similarly with insulin, only the combination of metformin with insulin seems to cause that patients demonstrate the significantly reduced LOX-1 serum level. This is a very important finding that can help geriatricians make therapeutic decisions.

Free amine groups are potentially available to react with a reducing sugar, therefore, this is an important parameter for us regarding the potential for glycation. In addition, Valencia et al. found that free amine content measured using OPA correlates strongly with RAGE binding affinity to AGE [46]. Fracesso expected differences between the amount of free amine content in the serum and tissues of rats after myocardial infarction compared to controls but did not find them [25]. It seems that this is the first time when this is observed in geriatric patients. Higher levels of serum free amino groups were found in the study patients with ischemic heart disease and after stroke. These are diseases associated with atherosclerosis. We expect that if there were more people in the test group with history of myocardial infarction, we would also see a difference with the control group. Therefore, it can be concluded that patients with cardiovascular diseases have an increased level of free amine content in the serum, and thus a greater potential for glycation and probably greater binding affinity RAGE to AGE. To increase the credibility of these statements, the study should be repeated on a larger geriatric population.

In addition to free amine content, we also observe higher AGE and pentosidine values in patientswith ischemic heart disease. This was an expected result, as it was previously reported that circulating fluorescent AGE and pentosidine levels have been associated with cardiovascular disease [47-51]. The same applies to the relationship of pentosidine with arterial hypertension. These dependencies are so strong that IHD and GFR can be considered as a predictor of

Page 5/12 
fluorescent AGE content, and IHD and hypertension as a predictor of high pentosidine levels. These correlations have been described earlier [50, 52, 53]. This is a logical consequence of the fact that glycation causes the formation of cross-links between collagen fibers and other proteins responsible for biochemical properties in the arteries, making them more rigid, unable to dilate properly, and manifesting in hypertension [54].

It is stated that the kidney normally clears circulating AGE but these products accumulate both in diabetic and not diabetic nephropathy [55]. Both fluorescent AGEs and pentosidines correlate positively with creatinine and negatively with GFR in our study. A correlation similar to the one we found was reported by Dozio et al based on patients with chronic kidney disease [55], Yoshida based on patients who underwent renal transplantation [47] and Schalkwijk comparing hemodialysed patients with normal subjects [56]. We would like to point out that there were generally no subjects with renal failure in our study group, so one may wonder whether fluorescent AGE/ pentosidine is not an indicator showing subclinical renal changes.

We do not try to present pentosidine or fluorescent AGEs as a diagnostic tool but we want to pay attention to the low cost, speed and simplicity of AGE determination by measuring fluorescence compared to other methods, such as enzyme immunoassay or liquid chromatography with tandem mass spectrometry (LC-MS/MS). For research purposes, this method seems to be sufficient.

The glycation pathway is associated with reactive oxidative species (ROS) and with peroxidation. ROS peroxidise membrane unsaturated fatty acids, leading to generate reactive aldehydes as advanced lipid peroxidation end products (ALE). Resulting reactive aldehydes react with proteins to cause alteration of protein structure to exacerbate complication of diseases like diabetes and atherosclerosis [57]. One of the classic products of lipid peroxidation is malondialdehyde which is easily determined in serum and tissues [58]. For many years, laboratory animals and human studies have analyzed the effect of hypoglycemic therapies on oxidative stress and the intensity of lipid peroxidation. Depending on the research model, the results vary, for example, Sotoudeh showed in the study with diabetic rats that metformin reduces MDA levels [59], Kocer claimed the same while looking at the results of women with polycystic ovaries [58] yet Alrefai reported that metformin does not lower MDA in obese patients with T2DM [60]. Similarly, in the case of insulin, there have been reports of both an anti-inflammatory role [61] as well as that hyperinsulinemia seems to cause exaggerating oxidative stress [62]. Bunck et al. confirmed that insulin therapy alone for one year does not cause a decrease in MDA levels [63]. Many researchers recommend a combination therapy with insulin as the best therapeutic approach for reducing oxidative stress in patients who need glycemic control $[60,62,64]$. The results of our study on the group of geriatric patients confirm that treatment with both metformin and insulin causes a decrease in the concentration of the lipid peroxidation marker.

\section{Summary And Conclusions}

Currently, the only routinely tested diagnostic parameter related to glycation is glycated hemoglobin. We want to emphasize that $\mathrm{HbA} 1 \mathrm{c}$ is not the de facto product of advanced glycation, it is a product of Amadori rearrangement - what can be called an early glycation product [65]. In the present study, we propose a new approach by analyzing several glycation parameters: fluorescent glycation products, as well as four different types of receptors for AGEs and ancillary two parameters related to oxidative stress and peroxidation that accompany aging and glycation.

Glutathione and SR-A have been shown to be of no noticeable value as indicators of typical geriatric diseases but other parameters appear to be interestingly related to pathologies of old age. According to our study, fluorinated AGEs and pentosidine are significantly increased in ischemic heart disease and arterial hypertension, and are also associated with kidney function. We also found indications that sRAGE and SR-BI have anti-atherogenic effects. The role of galectin 3 and LOX-1 in the pathomechanism of senile diseases is still under investigation. In our study, we observed a difference in the concentration of these proteins depending on the treatment. Combined treatment with insulin and metformin seems to be particularly beneficial as it reduces the intensity of lipid peroxidation and probably influences the expression of the protein responsible for lipoprotein binding and thus has the potential to inhibit atherosclerosis. Metformin alone results in a decrease in galectin 3 , which may protect against fibrosis and organ failure, such as the kidneys and the heart.

As emphasized several times in the discussion, this research should be repeated on a larger geriatric population. However, this is likely a great value in this research direction due to the possibility of finding a parameter that allowed for an even earlier diagnosis of prediabetic conditions or other numerous diseases of old age, as well as the possibility of monitoring the treatment of metabolic diseases typical of the elderly.

\section{List Of Abbreviations}

AGEs- Advanced Glycation End Products

AIC- Akaike Information Criterion

ALE- Advanced Lipid Peroxidation End Products

ANOVA-Analysis of Variance

BMI- Body Mass Index

BSA- Bovine Serum Albumin

COVID-19- Coronavirus Disease 2019

CRP- C-Reactive Protein

DM- Diabetes Mellitus 
DTNB- 5.5'-DiThiobis-2-NitroBenzoic acid

ELISA- Enzyme-Linked ImmunoSorbent Assay

GAL3- Galectin 3

GFR- Gromelular Filtration Rate

GLM- Generalized Linear Model

HbA1c- glycated hemoglobin type A1c

HDL- High-Density Lipoprotein

HUVECs- Human Umbilical Vein Endothelial Cells

IHD- Ischemic Heart Disease

LC-MS/MS- Liquid Chromatography-with tandem Mass Spetrometry

LDL- Low-Density Lipoprotein

LOX-1- Lectin-Like OXidized Low-Density Lipoprotein Receptor-1

MDA-MalonDiAldehyde

MSR1- Macrophage Scavenger Receptor 1

oxLDL- oxidized Low-Density Lipoprotein

OPA- O-PhthalAldehyde

RAGE- Receptor for Advanced Glycation End Products

ROS- Reactive Oxygen Species

RPM- Revolutions Per Minute

SARS-CoV-2- Severe Acute Respiratory Syndrome coronavirus 2

SCARB1 - Scavenger receptor class B member 1 gene

SR-A- Scavenger Rreceptor class A

sRAGE- soluble Receptor for Advanced Glycation End Products

SR-BI -Scavenger Receptor class B type I

TBA- ThioBarbituric Acid

TCA- TriChloroacetic Acid

T2DM- type 2 Diabetes Mellitus

WHO- World Health Organisation

\section{Declarations}

Ethics approval and consent to participate

The study protocol was approved by the Medical Ethics Committee of Wroclaw Medical University (opinion number KB-344/2017) and was in accordance with the ethical standards formulated in the Helsinki Declaration of 1975. Informed consent was obtained from all subjects.

Consent for publication

Not applicable

Availability of data and materials

The datasets used and/or analysed during the current study are available from the corresponding author on reasonable request. 
Competing interests

The authors declare that they have no competing interests.

Funding

Not applicable

Authors' contributions

Study concept and design: A.K.; acquisition of subjects: K.L., M.S.; analysis and interpretation of data: A.K.; preparation of manuscript: A.K., E.K; supervision: I.K-W., M.S.

Acknowledgements

Not applicable

\section{References}

1. Nascher I.L. Geriatrics. NY Med J. 1909;21:358-9.

2. Nascher IL. Geriatrics: The diseases of old age and their treatment, including physiological old age, home and institutional care, and medico-legal relations. P. Blakiston's Son \& Company; 1914.

3. Derejczyk J, Bień B, Kokoszka-Paszkot J, Szczygieł J. Gerontologia i geriatria w Polsce na tle Europy - czy należy inwestować w ich rozwój w naszym kraju? Gerontol Pol. 2008;16:149-59.

4. Jaul E, Barron J. Age-Related Diseases and Clinical and Public Health Implications for the 85 Years Old and Over Population. Front Public Heal. 2017;5 December:1-7.

5. Leszko M, Zając-Lamparska L, Trempala J. Aging in Poland. Gerontologist. 2015;55:707-15. doi:10.1093/geront/gnu171.

6. Cybulski M, Krajewska-Kułak E, Sowa P, Shpakau A, Theodosopoulou E, Chadzopulu A. Most common health problems of elderly in the opinion of health sciences students in Poland, Belarus and Greece. Prog Heal Sci. 2016;6:39-45.

7. Bautmans I, Salimans L, Njemini R, Beyer I, Lieten S, Liberman K. The effects of exercise interventions on the inflammatory profile of older adults: A systematic review of the recent literature. Exp Gerontol. 2021;146:111236.

8. Chentli F, Azzoug S, Mahgoun S. Diabetes mellitus in elderly. Indian J Endocrinol Metab. 2015;19:744-52.

9. Sinclair A, Morley JE, Rodriguez-Mañas L, Paolisso G, Bayer T, Zeyfang A, et al. Diabetes mellitus in older people: position statement on behalf of the International Association of Gerontology and Geriatrics (IAGG), the European Diabetes Working Party for Older People (EDWPOP), and the International Task Force of Experts in Diabetes. J Am Med Dir Assoc. 2012;13:497-502.

10. Boyle JP, Thompson TJ, Gregg EW, Barker LE, Williamson DF. Projection of the year 2050 burden of diabetes in the US adult population: Dynamic modeling of incidence, mortality, and prediabetes prevalence. Popul Health Metr. 2010;8:1-12.

11. Gentile S, Strollo F, Ceriello A. COVID-19 infection in Italian people with diabetes: Lessons learned for our future (an experience to be used). Diabetes Research and Clinical Practice. 2020;162. doi:10.1016/j.diabres.2020.108137.

12. Cuschieri S, Grech S. COVID-19 and diabetes: The why, the what and the how. J Diabetes Complications. 2020;34:107637. doi:10.1016/j.jdiacomp.2020.107637.

13. Yanase T, Yanagita I, Muta K, Nawata H. Frailty in elderly diabetes patients. Endocr J. 2018;65:1-11.

14. Thompson KH, Godin D V. Micronutrients and antioxidants in the progression of diabetes. Nutr Res. 1995;15:1377-410.

15. Newsholme P, Cruzat VF, Keane KN, Carlessi R, de Bittencourt PIHJ. Molecular mechanisms of ROS production and oxidative stress in diabetes. Biochem J. 2016;473:4527-50.

16. Nowotny K, Jung T, Höhn A, Weber D, Grune T. Advanced glycation end products and oxidative stress in type 2 diabetes mellitus. Biomolecules. 2015;5:194-222.

17. Newlaczyl AU, Yu LG. Galectin-3 - A jack-of-all-trades in cancer. Cancer Letters. 2011;313:123-8.

18. Böhme R, Becker C, Keil B, Damm M, Rasch S, Beer S, et al. Serum levels of advanced glycation end products and their receptors sRAGE and Galectin-3 in chronic pancreatitis. Pancreatology. 2020;20:187-92.

19. Stephen SL, Freestone K, Dunn S, Twigg MW, Homer-Vanniasinkam S, Walker JH, et al. Scavenger Receptors and Their Potential as Therapeutic Targets in the Treatment of Cardiovascular Disease. Int J Hypertens. 2010;2010:1-21. doi:10.4061/2010/646929.

20. Neviere R, Yu Y, Wang L, Tessier F, Boulanger E. Implication of advanced glycation end products (Ages) and their receptor (Rage) on myocardial contractile and mitochondrial functions. Glycoconj J. 2016;33:607-17. doi:10.1007/s10719-016-9679-x.

21. Hamasaki S, Kobori T, Yamazaki Y, Kitaura A, Niwa A, Nishinaka T, et al. Effects of scavenger receptors-1 class A stimulation on macrophage morphology and highly modified advanced glycation end product-protein phagocytosis. Sci Rep. 2018;8:1-11. doi:10.1038/s41598-018-24325-y.

22. Leszek J, Małyszczak K, Bartyś A, Staniszewska M, Gamian A. Analysis of Serum of Patients With Alzheimer's Disease for the Level of Advanced Glycation End Products. Am J Alzheimer's Dis Other Dementiasr. 2006;21:360-5. doi:10.1177/1533317506291075.

23. Diplock AT, Symons MCR, Rice-Evans CA. Techniques in free radical research. Elsevier; 1991. 
24. Shetty S, Babu S, Kumari S, Shetty P, Hegde S, Castelino R. Status of salivary lipid peroxidation in oral cancer and precancer. Indian J Med Paediatr Oncol. 2014;35:156-8. doi:10.4103/0971-5851.138990.

25. Fracasso B de M, Rangel JO, Machado AG, Curuja FS, Lopes A, Olsen V, et al. Characterization of advanced glycation end products and their receptor (RAGE) in an animal model of myocardial infarction. PLoS One. 2019;14:e0209964. doi:10.1371/journal.pone.0209964.

26. Hrynchyshyn N, Jourdain P, Desnos M, Diebold B, Funck F. Galectin-3: A new biomarker for the diagnosis, analysis and prognosis of acute and chronic heart failure. Archives of Cardiovascular Diseases. 2013;106:541-6.

27. Kanukurti J, Mohammed N, Sreedevi NN, Khan SA, Baba KSSS, Bhaskar MV, et al. Evaluation of Galectin-3 as a Novel Diagnostic Biomarker in Patients with Heart Failure with Preserved Ejection Fraction. J Lab Physicians. 2020;12:126-32. doi:10.1055/s-0040-1716608.

28. Atalar MN, Abuşoğlu S, Ünlü A, Tok O, İpekçi SH, Baldane S, et al. Assessment of serum galectin-3, methylated arginine and Hs-CRP levels in type 2 diabetes and prediabetes. Life Sci. 2019;231. doi:10.1016/j.Ifs.2019.116577.

29. King DR, Salako DC, Arthur-Bentil SK, Rubin AE, Italiya JB, Tan JS, et al. Relationship between novel inflammatory biomarker galectin-3 and depression symptom severity in a large community-based sample. J Affect Disord. 2021;281:384-9.

30. Pang J, Nguyen VT, Rhodes DH, Sullivan ME, Braunschweig C, Fantuzzi G. Relationship of galectin-3 with obesity, IL-6, and CRP in women. J Endocrinol Invest. 2016;39:1435-43. doi:10.1007/s40618-016-0515-8.

31. Zhang Z, Kang X, Guo Y, Zhang J, Xie J, Shao S, et al. Association of circulating galectin-3 with gestational diabetes mellitus, progesterone, and insulin resistance. J Diabetes. 2021;13:54-62. doi:10.1111/1753-0407.13088.

32. Alves MT, de Souza IDP, Ferreira CN, Cândido AL, Bizzi MF, Oliveira FR, et al. Galectin-3 is a potential biomarker to insulin resistance and obesity in women with polycystic ovary syndrome. Gynecol Endocrinol. 2020;36:760-3. doi:10.1080/09513590.2020.1739267.

33. Sattar Taha A, Zahraei Z, Al-Hakeim HK. Serum apelin and galectin-3 in preeclampsia in Iraq. Hypertens Pregnancy. 2020;39:379-86. doi:10.1080/10641955.2020.1777300.

34. Dikker O, Akarsu M. Evaluation of serum galectin-3 concentrations in patients with hypothyroidism. Scand J Clin Lab Invest. 2019;79:354-8. doi:10.1080/00365513.2019.1627576.

35. O'Seaghdha CM, Hwang SJ, Ho JE, Vasan RS, Levy D, Fox CS. Elevated galectin-3 precedes the development of CKD. J Am Soc Nephrol. 2013;24:1470-7. doi:10.1681/ASN.2012090909.

36. Tang WHW, Shrestha K, Shao Z, Borowski AG, Troughton RW, Thomas JD, et al. Usefulness of plasma galectin-3 levels in systolic heart failure to predict renal insufficiency and survival. Am J Cardiol. 2011;108:385-90. doi:10.1016/j.amjcard.2011.03.056.

37. Asensio-Lopez M del C, Lax A, Fernandez del Palacio MJ, Sassi Y, Hajjar RJ, Pascual-Figal DA. Pharmacological inhibition of the mitochondrial NADPH oxidase 4/PKCa/Gal-3 pathway reduces left ventricular fibrosis following myocardial infarction. Transl Res. 2018;199:4-23.

38. Marsche G, Weigle B, Sattler W, Malle E. Soluble RAGE blocks scavenger receptor CD36-mediated uptake of hypochlorite-modified low-density lipoprotein. FASEB J. 2007;21:3075-82. doi:10.1096/fj.07-8316com.

39. Barreto J, Karathanasis SK, Remaley A, Sposito AC. Role of LOX-1 (Lectin-Like Oxidized Low-Density Lipoprotein Receptor 1) as a Cardiovascular Risk Predictor: Mechanistic Insight and Potential Clinical Use. Arteriosclerosis, Thrombosis, and Vascular Biology. 2020;41:153-66. doi:10.1161/ATVBAHA.120.315421.

40. Akhmedov A, Sawamura T, Chen C-H, Kraler S, Vdovenko D, Lüscher TF. Lectin-like oxidized low-density lipoprotein receptor-1 (LOX-1): a crucial driver of atherosclerotic cardiovascular disease. Eur Heart J. 2020. doi:10.1093/eurheartj/ehaa770.

41. Mentrup T, Cabrera-Cabrera F, Schröder B. Proteolytic Regulation of the Lectin-Like Oxidized Lipoprotein Receptor LOX-1. Front Cardiovasc Med. 2021;7. doi:10.3389/fcrm.2020.594441.

42. West M, Greason E, Kolmakova A, Jahangiri A, Asztalos B, Pollin TI, et al. Scavenger receptor class B type i protein as an independent predictor of highdensity lipoprotein cholesterol levels in subjects with hyperalphalipoproteinemia. J Clin Endocrinol Metab. 2009;94:1451-7. doi:10.1210/jc.2008-1223.

43. Hung CH, Chan SH, Chu PM, Lin HC, Tsai KL. Metformin regulates oxLDL-facilitated endothelial dysfunction by modulation of SIRT1 through repressing LOX-1-modulated oxidative signaling. Oncotarget. 2016;7:10773-87. doi:10.18632/oncotarget.7387.

44. Shiu SWM, Wong Y, Tan KCB. Effect of advanced glycation end products on lectin-like oxidized low density lipoprotein receptor-1 expression in endothelial cells. J Atheroscler Thromb. 2012;19:1083-92. doi:10.5551/jat.11742.

45. Ouslimani N, Mahrouf M, Peynet J, Bonnefont-Rousselot D, Cosson C, Legrand A, et al. Metformin reduces endothelial cell expression of both the receptor for advanced glycation end products and lectin-like oxidized receptor 1. Metabolism. 2007;56:308-13. doi:10.1016/j.metabol.2006.10.010.

46. Valencia J V., Weldon SC, Quinn D, Kiers GH, DeGroot J, TeKoppele JM, et al. Advanced glycation end product ligands for the receptor for advanced glycation end products: Biochemical characterization and formation kinetics. Anal Biochem. 2004;324:68-78.

47. Yoshida N, Okumura Kl, Aso Y. High serum pentosidine concentrations are associated with increased arterial stiffness and thickness in patients with type 2 diabetes. Metabolism. 2005;54:345-50. doi:10.1016/j.metabol.2004.09.014.

48. Guerin-Dubourg A, Cournot M, Planesse C, Debussche X, Meilhac O, Rondeau P, et al. Association between Fluorescent Advanced Glycation End-Products and Vascular Complications in Type 2 Diabetic Patients. Biomed Res Int. 2017;2017. doi:10.1155/2017/7989180.

49. Bodiga VL, Eda SR, Bodiga S. Advanced glycation end products: Role in pathology of diabetic cardiomyopathy. Heart Failure Reviews. 2014;19:49-63. doi:10.1007/s10741-013-9374-y.

50. Sugiyama S, Miyata T, Ueda Y, Tanaka H, Maeda K, Kawashima S, et al. Plasma levels of pentosidine in diabetic patients: an advanced glycation end product. J Am Soc Nephrol. 1998;9. 
51. Kerkeni M, Weiss IS anto., Jaisson S, Dandana A, Addad F, Gillery P, et al. Increased serum concentrations of pentosidine are related to presence and severity of coronary artery disease. Thromb Res. 2014;134:633-8. doi:10.1016/j.thromres.2014.07.008.

52. Kerkeni M, Saïdi A, Bouzidi H, Letaief A, Ben Yahia S, Hammami M. Pentosidine as a biomarker for microvascular complications in type 2 diabetic patients. Diabetes Vasc Dis Res. 2013;10:239-45. doi:10.1177/1479164112460253.

53. Nakano M, Nakamura Y, Suzuki T, Miyazaki A, Takahashi J, Saito M, et al. Pentosidine and carboxymethyl-lysine associate differently with prevalent osteoporotic vertebral fracture and various bone markers. Sci Rep. 2020;10:22090. doi:10.1038/s41598-020-78993-W.

54. Munenori K, Kitazawa R, Makita K, Yoshida K, Takeji M, Soga Y, et al. Pulmonary hypertension associated with diffuse deposition of pentosidine in pulmonary arterioles. Diabetes Res Clin Pract. 2013;100. doi:10.1016/j.diabres.2013.01.019.

55. Dozio E, Vettoretti S, Caldiroli L, Nerini-Molteni S, Tacchini L, Ambrogi F, et al. Advanced glycation end products (Age) and soluble forms of age receptor: Emerging role as mortality risk factors in CKD. Biomedicines. 2020;8:1-12. doi:10.3390/biomedicines8120638.

56. Schalkwijk CG, Ter Wee PM, Stehouwer CDA. Plasma levels of AGE peptides in type 1 diabetic patients are associated with serum creatinine and not with albumin excretion rate: Possible role of AGE peptide-associated endothelial dysfunction. In: Annals of the New York Academy of Sciences. New York Academy of Sciences; 2005. p. 662-70. doi:10.1196/annals.1333.075.

57. Nooshi-Nedamani S, Habibi-Rezaei M, Farzadfard A, Moosavi-Movahedi AA. Intensification of serum albumin amyloidogenesis by a glycationperoxidation loop (GPL). Arch Biochem Biophys. 2019;668:54-60. doi:10.1016/j.abb.2019.05.008.

58. Kocer D, Bayram F, Diri H. The effects of metformin on endothelial dysfunction, lipid metabolism and oxidative stress in women with polycystic ovary syndrome. Gynecol Endocrinol. 2014;30:367-71. doi:10.3109/09513590.2014.887063.

59. Sotoudeh R, Hadjzadeh M-A-R, Gholamnezhad Z, Aghaei A. The anti-diabetic and antioxidant effects of a combination of Commiphora mukul, Commiphora myrrha and Terminalia chebula in diabetic rats. Avicenna J phytomedicine. 2019;9:454-64. doi:10.22038/ajp.2019.12721.

60. ALrefai AA, Alsalamony AM, Fatani SH, Kamel HFM. Effect of variable antidiabetic treatments strategy on oxidative stress markers in obese patients with T2DM. Diabetol Metab Syndr. 2017;9. doi:10.1186/s13098-017-0220-6.

61. Sun Q, Li J, Gao F. New insights into insulin: The anti-inflammatory effect and its clinical relevance. World J Diabetes. 2014;5:89. doi:10.4239/wjd.v5.i2.89.

62. Monnier L, Colette C, Michel F, Cristol JP, Owens DR. Insulin therapy has a complex relationship with measure of oxidative stress in type 2 diabetes: A case for further study. Diabetes Metab Res Rev. 2011;27:348-53. doi:10.1002/dmrr.1174.

63. Bunck MC, Cornér A, Eliasson B, Heine RJ, Shaginian RM, Wu Y, et al. One-year treatment with exenatide vs. Insulin Glargine: Effects on postprandial glycemia, lipid profiles, and oxidative stress. Atherosclerosis. 2010;212:223-9.

64. Zhang X, Liu Y, Xiong D, Xie C. Insulin combined with Chinese medicine improves glycemic outcome through multiple pathways in patients with type 2 diabetes mellitus. J Diabetes Investig. 2015;6:708-15. doi:10.1111/jdi.12352.

65. Zhang L, Zhang Q. Glycated Plasma Proteins as More Sensitive Markers for Glycemic Control in Type 1 Diabetes. PROTEOMICS - Clin Appl. 2020;14:1900104. doi:10.1002/prca.201900104.

\section{Tables}

Table 1. Characteristics of the study group

\begin{tabular}{|c|c|c|c|c|c|}
\hline & Without diabetes $0(\mathrm{~N}=38)$ & With diabetes $1(\mathrm{~N}=41)$ & $\mathbf{p}$ & & Statistical test \\
\hline AGE (Max-Min) & $79,65(64,0-92,0)$ & $78,7(64,0-94,0)$ & 0,52 & & Mann-Whitney U test \\
\hline $\operatorname{Sex}(K / M)$ & $29 / 9$ & $30 / 11$ & 0,7480 & & $\chi 2$ test \\
\hline BMI (Max-Min) & $26,21(17,4-46,6)$ & $29,43(18,7-38,6)$ & 0,003 & * & Mann-Whitney U test \\
\hline ischemic heart disease $0 / 1$ & $27 / 9$ & $25 / 12$ & 0,4831 & & $\chi 2$ test \\
\hline Myocardial infarction $0 / 1$ & $37 / 1$ & $35 / 6$ & 0,0667 & & Fisher's exact test \\
\hline Stroke 0/1 & $32 / 5$ & $36 / 5$ & 0,5634 & & Fisher's exact test \\
\hline Hypertension 0/1 & $11 / 27$ & $6 / 35$ & 0,1014 & & Fisher's exact test \\
\hline Peripheral artery disease $0 / 1$ & $34 / 3$ & $37 / 4$ & 0,5582 & & Fisher's exact test \\
\hline Atherosclerosis $0 / 1$ & $13 / 25$ & $9 / 32$ & 0,2245 & & $\chi 2$ test \\
\hline Hyperlipidemia 0/1 & $18 / 19$ & $28 / 12$ & 0,0563 & & $\chi 2$ test \\
\hline fatty liver $0 / 1$ & $32 / 6$ & $30 / 11$ & 0,2329 & & $\chi 2$ test \\
\hline
\end{tabular}

Table 2. Correlation matrix between the analyzed parameters. 


\begin{tabular}{|c|c|c|c|c|c|c|c|c|c|c|c|c|}
\hline & AGE & Pentosidine & $\begin{array}{l}\text { Free } \\
\text { amine } \\
\text { content }\end{array}$ & GAL3 & LOX-1 & sRAGE & BMI & HbA1C & Glucose & Cholesterol & HDL & LDL \\
\hline Pentosidine & 0,6728 & & & & & & & & & & & \\
\hline $\begin{array}{l}\text { Free amine } \\
\text { content }\end{array}$ & 0,0469 & 0,116 & & & & & & & & & & \\
\hline GAL3 & 0,0015 & $-0,0395$ & 0,0075 & & & & & & & & & \\
\hline LOX-1 & $-0,2225$ & $-0,2296$ & 0,0886 & $-0,0459$ & & & & & & & & \\
\hline sRAGE & 0,0052 & 0,0172 & $-0,0254$ & 0,2726 & 0,2683 & & & & & & & \\
\hline BMI & $-0,0868$ & $-0,1671$ & 0,0183 & 0,6198 & 0,0432 & 0,0205 & & & & & & \\
\hline $\mathrm{HbA1C}$ & 0,1603 & 0,2302 & 0,0416 & 0,2675 & $-0,1339$ & 0,2283 & 0,281 & & & & & \\
\hline Glucose & $-0,0571$ & $-0,124$ & 0,0368 & 0,3888 & 0,1228 & 0,1911 & 0,3447 & 0,6508 & & & & \\
\hline Cholesterol & 0,1864 & 0,1493 & 0,0908 & 0,1582 & $-0,1727$ & 0,1135 & 0,1517 & $-0,1073$ & $-0,1188$ & & & \\
\hline HDL & 0,0611 & 0,1746 & 0,0763 & $-0,0342$ & $-0,1656$ & $-0,292$ & $-0,1984$ & $-0,2548$ & $-0,2409$ & 0,4286 & & \\
\hline LDL & 0,1671 & 0,0758 & 0,0442 & 0,1233 & $-0,1496$ & 0,1111 & 0,1163 & $-0,2332$ & $-0,2119$ & 0,9639 & 0,2839 & \\
\hline Triglycerides & 0,1255 & 0,1085 & 0,2192 & 0,3631 & 0,026 & 0,4673 & 0,4121 & 0,2145 & 0,2169 & 0,3639 & $-0,2112$ & 0,2931 \\
\hline Creatinine & 0,4553 & 0,258 & 0,125 & 0,1413 & 0,0963 & 0,2113 & 0,1453 & 0,0646 & 0,0857 & $-0,0668$ & $-0,3561$ & $-0,0719$ \\
\hline GFR & $-0,4853$ & $-0,3551$ & $-0,1351$ & $-0,3204$ & $-0,0864$ & $-0,3593$ & $-0,1741$ & $-0,1169$ & $-0,0793$ & $-0,113$ & 0,2475 & $-0,1076$ \\
\hline Uric acid & $-0,1098$ & $-0,154$ & 0,3177 & 0,2348 & 0,1995 & 0,0812 & 0,3038 & 0,0676 & 0,176 & $-0,0546$ & $-0,2416$ & $-0,0427$ \\
\hline SR-BI & $-0,011$ & $-0,2438$ & 0,1062 & 0,0286 & 0,3121 & 0,4733 & $-0,0441$ & 0,0757 & 0,1545 & $-0,1323$ & $-0,2987$ & $-0,0857$ \\
\hline SR-A & 0,2534 & $-0,0282$ & 0,043 & $-0,0569$ & 0,0273 & $-0,0623$ & 0,1263 & 0,2229 & $-0,0017$ & 0,1264 & 0,0181 & 0,1616 \\
\hline
\end{tabular}

\section{Figures}
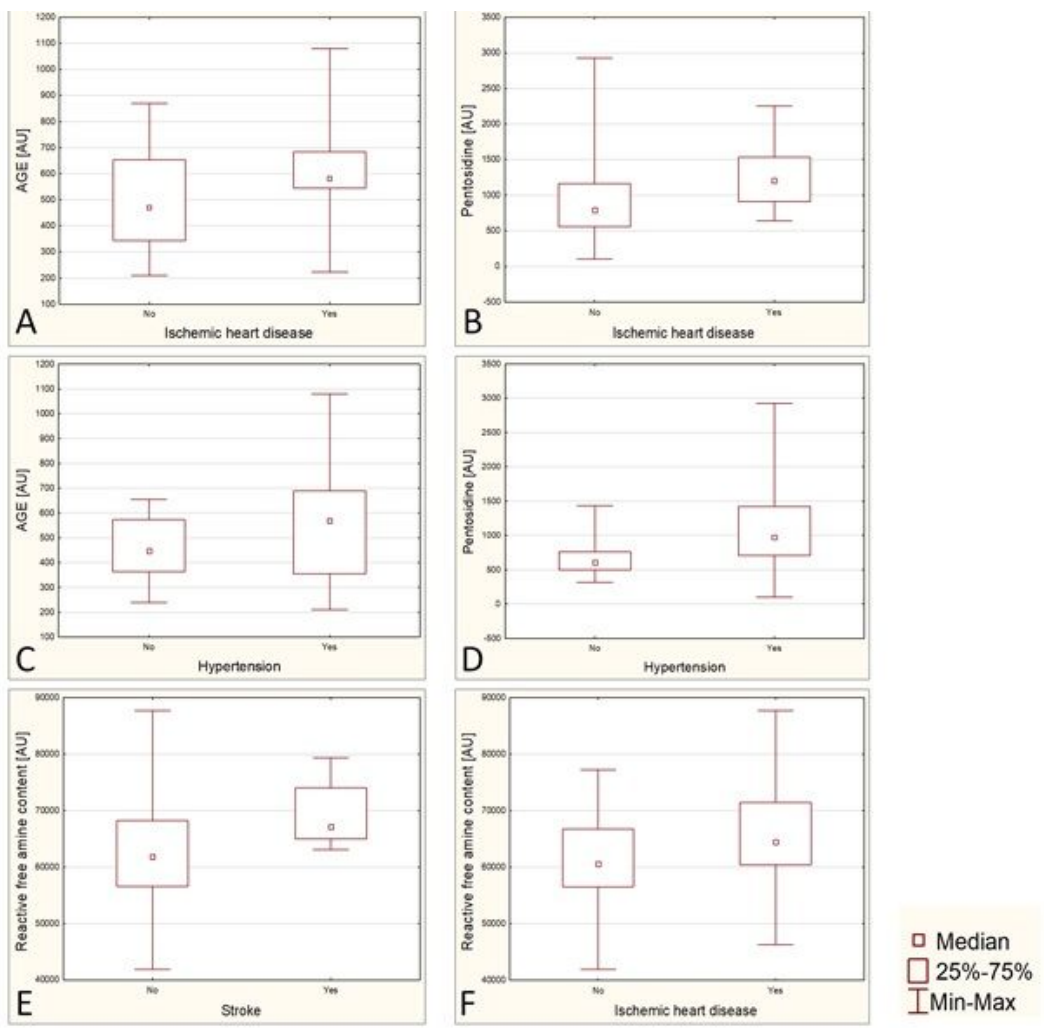

Figure 1

Page $11 / 12$ 
Group comparison between variables (box and whisker plots). Comparison of AGE levels (panel A), pentosidine (panel B) and reactive free amine conntent (panel F) in patients with and without ischemic heart disease. Comparison of AGE (panel C) and pentosidine (panel D) in with and without hypertension. Comparing the level of reactive free amine content in patients with and without stroke (panel E).

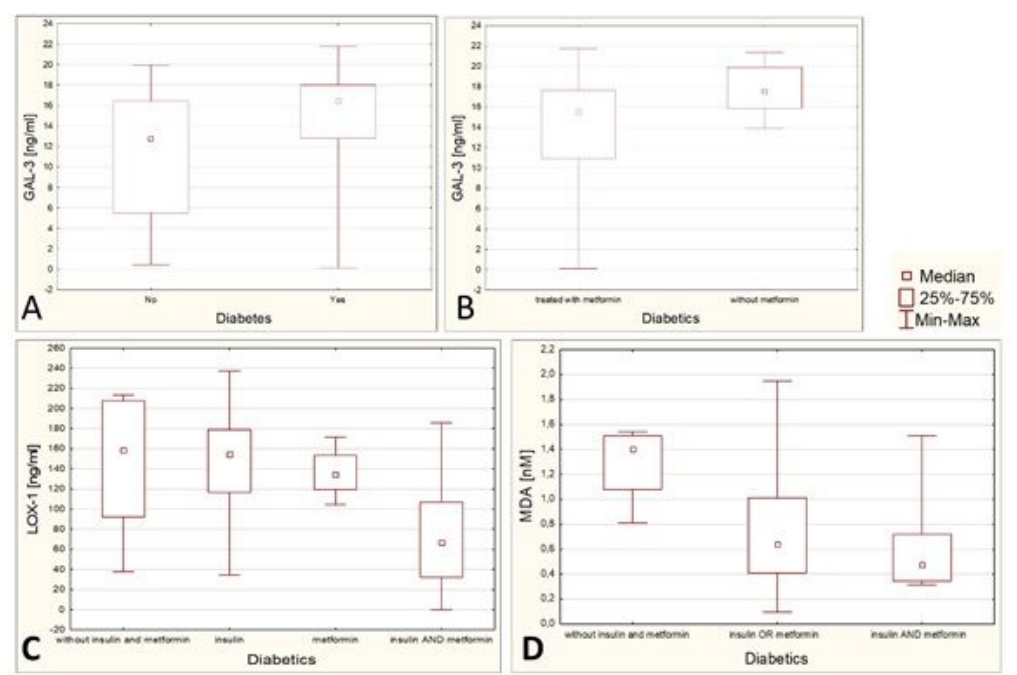

Figure 2

Group comparison between variables (box and whisker plots). Comparison of GAL3 levels in patients with and without diabetes (panel A). Comparison of GAL3 levels in patients taking and not taking metformin (panel B). Comparison of LOX-1 levels in diabetic patients not treated with insulin or metformin, treated with insulin, metformin or both medications simultaneously (panel C). Comparison of MDA levels between diabetic patients not treated with insulin or metformin, treated with insulin or metformin and both medications simultaneously (panel D). 